\title{
Approaching Neoadjuvant Therapy in the Management of Early-Stage Breast Cancer
}

This article was published in the following Dove Press journal:

Breast Cancer: Targets and Therapy

\author{
Tara Hyder' \\ Saveri Bhattacharya ${ }^{2, *}$ \\ Kristine Gade ${ }^{3, *}$ \\ Azadeh Nasrazadani ${ }^{3}$ \\ Adam M Brufsky \\ 'University of Pittsburgh Physicians, \\ Pittsburgh, PA, USA; ${ }^{2}$ Department of \\ Medical Oncology, Sidney Kimmel Cancer \\ Center, Thomas Jefferson University, \\ Philadelphia, PA, USA; ${ }^{3}$ UPMC Hillman \\ Cancer Center, Magee Women's \\ Hospital, Pittsburgh, PA, USA \\ *These authors contributed equally to \\ this work
}

\begin{abstract}
Neoadjuvant therapy is integral to the treatment of early-stage breast cancer. Goals of treatment include surgical downstaging of the tumor, rendering inoperable tumors resectable, and de-escalating axillary surgery in those with clinically positive nodes. Additionally, response to treatment provides important prognostic information regarding risk of recurrence and guides future adjuvant treatment. Although chemotherapy serves as the backbone of neoadjuvant treatment, an increased understanding of the tumor's clinical course as well as its molecular and genetic make-up aids in individualizing treatment and developing novel agents. This review summarizes current clinical approaches and the future direction to the management of breast cancer patients in the neoadjuvant setting.
\end{abstract}

Keywords: neoadjuvant chemotherapy, neoadjuvant endocrine therapy, pCR, breastconserving surgery

\section{Introduction}

Breast cancer is the most frequently diagnosed cancer and the leading cause of cancerrelated death in women globally. ${ }^{1}$ Treatment decisions are tailored based on the size of breast cancer, degree of lymph node involvement, expression of the estrogen receptor (ER) and progesterone receptor (PR), and expression of the HER2 protein. Neoadjuvant therapy refers to the administration of systemic therapy, either chemotherapy or endocrine therapy, prior to definitive breast surgery. Patients who derive clinical benefit from neoadjuvant chemotherapy (NACT) include patients with high-risk breast tumors, large breast tumors, and locally advanced tumors, including those initially ineligible for surgery. The goals of NACT include rendering inoperable tumors resectable, surgical downstaging for patients who prefer breast conservation, and deescalating axillary surgery in those with clinically positive nodes.

\section{Rationale for Neoadjuvant Systemic Therapy Compared to Adjuvant Therapy}

Early initiation of systemic therapy was thought to improve overall survival (OS) in high-risk patients receiving NACT. However, in the Early Breast Cancer Trialists' Collaborative Group (EBCTCG) meta-analysis, no difference was observed in distant recurrence rate $(38.2 \%$ vs $38 \%$, RR $1.02,0.92-1.14)$ or breast cancer mortality $(34.4 \%$ vs $33.7 \%$, RR $1.06,0.95-1.18$ ) in patients treated with NACT compared to adjuvant chemotherapy at 15-year follow-up. ${ }^{2}$ Furthermore, despite NACT leading to a higher frequency of breast-conserving therapy (BCT) (65\% vs 49\%) compared to adjuvant chemotherapy, there was also an associated increased risk of local recurrence in
Correspondence: Adam M Brufsky UPMC Hillman Cancer Center, Magee Women's Hospital, Suite 4628, 300 Halket Street, Pittsburgh, PA, I52/3, USA

Email brufskyam@upmc.edu 
patients (21.4\% vs $15.9 \%$; RR 1.37, 1.17-1.61). Similarly, the NSABP B-18 study showed equivalent disease-free survival (DFS), distant disease-free survival, and OS in patients receiving pre-operative and post-operative chemotherapy (OS; $\mathrm{p}=0.99,0.70$, and 0.83 , respectively), however with more women able to achieve BCT in the pre-operative chemotherapy group. ${ }^{3}$ One group that did experience a superior DFS and OS in those receiving NACT was patients who had a pathologic complete response (pCR). Altogether, the aforementioned studies suggest NACT provides therapy that is equivalent to adjuvant therapy and notably demonstrates a survival advantage in those who have a complete response. ${ }^{4}$

\section{Association of Pathological Complete Response with Survival Outcomes}

Response to neoadjuvant therapy provides important prognostic information and helps guide adjuvant therapy recommendations. It allows for an in vivo human model system to explore the efficacy of chemotherapy and leads to a better understanding of breast cancer biology. $\mathrm{pCR}$, as defined by the FDA, is the absence of residual invasive cancer on hematoxylin and eosin evaluation of the complete resected breast specimen and all sampled regional lymph nodes following completion of NACT (ypT0/is ypN0). ${ }^{5} \mathrm{pCR}$ has been shown to correlate with improved survival as demonstrated in the CTNeoBC pooled analysis. ${ }^{6}$ This pooled analysis of 12 international trials and 11,955 patients demonstrated improved event-free survival (EFS) and OS with ypT0/Tis ypN0 pCR with HR of 0.48 and 0.36 , respectively. The association with pCR and long-term outcomes was strongest in triple-negative breast cancer (TNBC) patients (EFS HR 0.24, OS HR 0.16) and HER2-positive, hormone-receptor negative patients who received trastuzumab (EFS HR 0.15, OS HR 0.08). Several other trials have also demonstrated that the subset of ERnegative and HER2-positive breast cancers who achieved a pCR after NACT have a notable survival advantage when compared to those with residual disease..$^{78}$ The presence of residual disease after NACT portends an increased risk of recurrence and further adjuvant therapy can be given to patients with HER2-positive or triple-negative breast cancer to prevent distant disease recurrence.

\section{Clinical and Radiological Assessment in the Neoadjuvant Approach}

Neoadjuvant chemotherapy allows for visualization of a marked reduction in tumor size, thus it is critical to accurately mark the primary tumor at the time of diagnosis. Prior to undergoing NACT, the following is required: physical examination, breast imaging including ultrasound, mammogram, and core biopsy, and a biopsy of the lymph node with clip/marker placement. Prior to each cycle of chemotherapy, a physical examination should be performed to monitor response to therapy. There are rare occasions where the tumor has little response or grows despite NACT, necessitating a change in therapy or proceeding to earlier surgery. Therefore, periodic physical examinations are crucial. At the conclusion of therapy, repeat breast imaging including a mammogram and ultrasound are performed. The following will discuss the key indications for neoadjuvant therapy (Table 1).

\section{Hormone Receptor-Positive Breast Cancer}

Hormone receptor (HR)-positive breast cancer accounts for approximately $75 \%$ of new breast cancer diagnoses. ${ }^{9}$ Most frequently, these patients will receive cytotoxic chemotherapy for their neoadjuvant treatment, although there are situations where NET is preferred over NACT. While NACT confers the greatest advantage in HER2-positive breast cancer and TNBC, the rates of $\mathrm{pCR}$ are lower in luminal cancer, averaging $10-20 \%$ of cases. ${ }^{5,8}$ This suggests that luminal cancers are less sensitive to chemotherapy compared to other subtypes and a potential alternative to cytotoxic chemotherapy is NET. A meta-analysis of 20 studies involving 3490 patients compared combination chemotherapy to NET with aromatase inhibitors (AI). ${ }^{10}$ Although both groups had a similar clinical response rate (OR, 1.08; 95\% CI, 0.50-2.35; $\mathrm{P}=0.85$ ), radiological

Table I Indications for Consideration of Neoadjuvant Chemotherapy

\begin{tabular}{|l|}
\hline *Hormone-receptor positive breast cancer \\
\hline High-risk HER2-positive breast cancer \\
\hline High-risk triple-negative breast cancer \\
\hline Inflammatory breast cancer \\
\hline Locally advanced tumors breast cancers \\
\hline Clinically node-positive breast cancers \\
\hline $\begin{array}{l}\text { Patients with contraindications to surgery including pregnancy or } \\
\text { venous thromboembolism (VTE) }\end{array}$ \\
\hline $\begin{array}{l}\text { Delayed elective surgical plans due to pandemic or other elective } \\
\text { procedures }\end{array}$ \\
\hline
\end{tabular}

Note: *In certain circumstances. 
response rate $(\mathrm{OR}, 1.38 ; 95 \% \mathrm{CI}, 0.92-2.07 ; \mathrm{P}=0.12)$, and $\mathrm{BCS}$ rate $(\mathrm{OR}, 0.65 ; 95 \% \mathrm{CI}, 0.41-1.03 ; \mathrm{P}=0.07)$, there were lower toxicities associated with AI monotherapy. These results suggest that in the face of similar outcomes, NET may be a preferred option in light of increased tolerability.

Tamoxifen is a long-standing treatment for HR-positive breast cancer. However, issues including tamoxifen resistance and serious adverse effects such as thromboembolism and endometrial cancer necessitate the use of other viable options. While earlier trials utilized tamoxifen, recent studies demonstrate better outcomes with $\mathrm{AI}$ in postmenopausal women. The P024 trial is the largest trial comparing neoadjuvant tamoxifen and letrozole in HRpositive postmenopausal women who were ineligible for breast-conserving surgery (BCS). Letrozole led to a significantly greater objective response rate (ORR) (55\% vs $36 \%, \mathrm{p}<0.0010)$ and rate of $\mathrm{BCS}(45 \%$ vs $35 \%$, $\mathrm{p}=0.022$ ) compared to tamoxifen. ${ }^{11}$ Both the PROACT and IMPACT trial compared neoadjuvant tamoxifen and anastrozole. $^{12,13}$ In the PROACT trial, anastrozole was superior to tamoxifen $(36.6 \%$ vs $24.4 \% \mathrm{p}=0.04)$ based on objective response measured by ultrasound evaluation. Although there was no difference in ORR between both arms in the IMPACT trial, among patients who were not BCS candidates at study entry, treatment with anastrozole resulted in increased $\mathrm{BCS}$ rate compared to the tamoxifen $\operatorname{arm}(46 \%$ vs $22 \% ; \mathrm{p}=0.03)$. Based on these enhanced outcomes with AI compared to tamoxifen, the ACOSOG Z1031 trial was designed to evaluate clinical outcomes among the AIs exemestane, letrozole, and anastrozole. ${ }^{14}$ In terms of ORR and BCS, there was no significant difference found between the different types of AIs, suggesting they are largely interchangeable.

Despite the advantages of neoadjuvant AI therapy, there are no clear guidelines about the duration of treatment. Although most NET randomized trials have a treatment duration of 3 to 4 months based on studies of tamoxifen and chemotherapy, there is concern that this might not be sufficient to achieve optimal tumor shrinkage. ${ }^{15,16}$ In clinical studies involving the administration of 12 months of NET, the ORRs ranges from 76.8 to $95 \%$, and the rate of BCS was 45 to $87.5 \% .^{15,17}$ In contrast, the PROACT and IMPACT trials used a duration of 3 months and reported an ORR of $49.7 \%$ and $37 \%$, respectively, and BCS rates of $43 \%$ and $44 \%$, respectively. ${ }^{12,13}$ Similar results were observed in a study utilizing exemestane therapy that evaluated tumor size at 3 and 6 months. ${ }^{18}$
Overall response was $58.7 \%$ at 3 months and $68.3 \%$ at final clinical palpation $(\mathrm{p}=0.001)$. The BCS rate increased from $61.8 \%$ to $70.6 \%(p=0.012)$. Although these studies demonstrate that long-term NET achieves further tumor reduction and increases $\mathrm{BCS}$ rates, there are concerns that prolonging NET until maximal response increases the risk of disease progression. Further studies will need to be done to investigate the optimal duration of NET and whether outcomes are not jeopardized as a result of chemotherapy exclusion.

Another area of interest is identifying reliable biomarkers for optimal patient selection and measuring treatment response to NET. Although pCR rates have been the standard to measure efficacy of neoadjuvant therapy, they may not be as reliable an outcome measure in HR-positive tumors. This has led to the development of the preoperative endocrine prognostic index (PEPI) which combines Ki-67 level, ER status, pathological tumor size, and node status in the surgical specimen following NET. ${ }^{19}$ Patients with a PEPI score of 0 have an extremely low risk of relapse and can be exempt from adjuvant chemotherapy. Scores of $0,1-3$, and $\geq 4$ corresponded to a risk of relapse of 10,23 , and $48 \%$ respectively. The PEPI score was further validated in the ACOSOG Z1031 trial. Data at a median follow-up time of 5.5 years showed a clear difference in relapse-free survival with $3.7 \%$ of patients with $\mathrm{PEPI}=0$ relapsing compared to $15 \%$ with $\mathrm{PEPI}>1$ (recurrence hazard ratio $[\mathrm{PEPI}=0$ vs $\mathrm{PEPI}>0]=0.35$; $p=0.14 ; 95 \% \mathrm{CI}, 0.092$ to 0.764$).{ }^{20}$ The ALTERNATE trial aims to assess the validity of Ki-67 level measurement following four-week treatments with anastrozole, fulvestrant, or the combination. After the initial treatment, women with a $\mathrm{Ki}-67>10 \%$ will be switched to NACT, while women with a $\mathrm{PEPI}=0$ will proceed to receive adjuvant endocrine therapy. ${ }^{21}$ Between all the treatment arms, no discernable differences were reported in endocrinesensitive disease rates (ESDR) or rate of breastconserving surgeries, although recurrence-free survival data are still pending.

Other novel agents are being evaluated with regard to their ability to improve the efficacy of NET or to overcome de novo resistance to NET. One such class of drugs is CDK4/6 inhibitors (CDKI) that have been shown to enhance the activity of AI in advanced disease. In the neoMONARCH and PALLET trials, the combination of NET with a CDKI (abemaciclib and ribociclib, respectively) resulted in decreased levels of $\mathrm{Ki}-67$, although this did not correspond to a significant improvement in 
clinical response rate in the PALLET trial. ${ }^{22,23}$ The CORALLEEN trial was a randomized Phase II trial investigating ribociclib plus letrozole vs chemotherapy in postmenopausal women with luminal B HR-positive breast cancer. $^{24}$ The proportion of patients achieving a PEPI of 0 was $22.4 \%$ with ribociclib and letrozole and $17.3 \%$ with chemotherapy, demonstrating the efficacy of NET combined with CDKIs in luminal B patients as well. Further research is needed to elucidate the potential role of CDKIs in the neoadjuvant setting.

The eventual development of endocrine resistance with endocrine therapy has led to increased interest in phosphatidylinositol 3-kinase (PI3K) inhibitors, including alpelisib and taselisib, due to the involvement of PI3K-AKT-mTOR pathway upregulation in endocrine resistance. The LORELEI trial is a phase II trial randomizing 334 patients with HR-positive breast cancer to neoadjuvant therapy with letrozole plus taselib or letrozole alone. The addition of taselisib to letrozole was associated with a higher proportion of patients achieving an ORR compared to letrozole alone $(50 \%$ vs $39 \%, \mathrm{p}=0.049) .{ }^{25}$ These results suggest that PI3K inhibitors do have a role in enhancing the effectiveness of NET in HR-positive breast cancer.

Another novel therapeutic approach involves immunotherapy, which has demonstrated promising results in the neoadjuvant treatment of TNBC. Checkmate 7FL (NCT04109066) is an ongoing Phase 3 study evaluating nivolumab versus placebo in combination with NACT. Patients will undergo surgery and receive either nivolumab or placebo with endocrine therapy in the adjuvant setting. ${ }^{26}$ Primary endpoints are pCR and event-free survival. KEYNOTE-756 is another phase 3 study utilizing neoadjuvant pembrolizumab (versus placebo) and chemotherapy followed by pembrolizumab (versus placebo) plus endocrine therapy in the adjuvant setting. ${ }^{27}$ However, to date, there are no data supporting a role for immunotherapy in the treatment of HR-positive tumors.

\section{High-Risk HER2-Positive Breast Cancer}

HER2-positive breast cancer is an aggressive subtype that is due to persistent activation of signaling of the human epidermal growth factor receptor 2 (HER2) pathway and accounts for $15 \%$ of all breast cancers. ${ }^{28}$ Patients with HER2-positive breast cancer who receive NACT have a higher rate of $\mathrm{pCR}$ with a combination of cytotoxic chemotherapy and targeted therapy compared to those who receive adjuvant chemotherapy. A 2016 metaanalysis of 5800 patients with HER2-positive breast cancer receiving NACT showed that those who achieved a pCR had an improved OS and event-free survival (EFS) compared to those who did not. ${ }^{29}$ Neoadjuvant chemotherapy for HER2-positive breast cancer includes administration of chemotherapy, either anthracycline based or non-anthracycline based, with anti-HER2 monoclonal antibodies (trastuzumab and pertuzumab). It remains controversial regarding the use of an anthracycline in the neoadjuvant setting for HER2-positive breast cancer.

Historically, anthracycline-based regimens have been used for treatment of HER2-positive breast cancer and are often reserved for high-risk subgroups such as those with lymph node involvement or a young age. In the NSABP B-41 study, patients with HER2-positive breast cancer were assigned to receive four cycles of doxorubicin and cyclophosphamide every three weeks followed by weekly paclitaxel-trastuzumab and were found to have an overall pCR rate of $49 \%$. In the lymph node-positive patients, the pCR rate was $43 \%{ }^{30}$

Subsequent studies have shown that treatment with an anthracycline is not critical. In the TRAIN-2 study, patients with early-stage HER2-positive breast cancer were treated with adjuvant chemotherapy, either an anthracyclinecontaining regimen or a non-anthracycline-based chemotherapy, combined with trastuzumab and pertuzumab. The pCR rate did not differ significantly between these two arms $(67 \%$ in the anthracycline arm vs $68 \%$ in the non-anthracycline arm), and the 3-year EFS (94\% vs 93\%), OS (98\% vs 98\%) were equivalent. ${ }^{31}$ No subgroup of patients benefited from the inclusion of an anthracycline and there were more side effects in the anthracycline-containing arm including higher rates of febrile neutropenia (10\% vs $1 \%$ ) and a significant decline in LVEF (36\% vs $22 \%$ ). In the randomized phase II TRYPHAENA study, the nonanthracycline-containing regimens plus trastuzumab and pertuzumab resulted in a pCR of $64 \%$ compared to a pCR of 55\% among those treated with an anthracycline-based regimen (FEC-THP) containing trastuzumab and pertuzumab and also resulted in lesser hematologic toxicity. ${ }^{32}$ Given the aforementioned studies, a taxane and carboplatin-containing regimen is a reasonable alternative to an anthracycline-containing regimen.

In addition to NACT, anti-HER2 therapy is routinely added, with trastuzumab representing the first targeted antiHER2 drug. In a meta-analysis of 2000 patients with HER2positive disease, the addition of trastuzumab to NACT 
increased the pCR from 23 to $40 \%{ }^{8}$ Similarly, in the phase II NOAH trial, patients treated with NACT and trastuzumab had an increase in pCR rate to $38 \%$ compared to $19 \%$ with NACT alone. ${ }^{33}$ Long-term follow-up demonstrated improved EFS in the group that received trastuzumab (43 vs $58 \%$; HR $0.64,95 \%$ CI $0.544-0.930) .{ }^{33}$ Despite the effectiveness of trastuzumab, there are clinical concerns about the development of acquired resistance over a period of time. In order to eradicate resistance, the addition of pertuzumab to trastuzumab has been investigated. In the Phase 2 NeoSphere trial, patients with locally advanced and inflammatory HER2positive breast cancer had a significantly improved pCR in the group receiving pertuzumab and trastuzumab plus docetaxel (45.8\%) compared to the other three groups who received trastuzumab plus docetaxel $(29 \%, \mathrm{p}=0.0141)$, pertuzumab plus trastuzumab (16.8\%), or pertuzumab and docetaxel $(24 \%){ }^{34}$ A meta-analysis of six trials involving approximately 2000 patients demonstrated an improved pCR rate of $16-19 \%$ in patients receiving dual blockade, irrespective of the chemotherapy backbone (RR 1.37, 95\% CI $1.23-1.53, p<0.0001) .{ }^{35}$ These findings suggest a role for the addition of pertuzumab to NACT and trastuzumab.

For patients with residual disease after neoadjuvant therapy, T-DM1 in the adjuvant setting can decrease the risk of recurrence. This recommendation is based on the results of the KATHERINE trial, in which 1486 HER2-positive patients with residual invasive disease after neoadjuvant therapy with a taxane and trastuzumab or dual HER2-blockade were randomized to receive adjuvant T-DM1 or trastuzumab. T-DM1 administration improved 3-year invasive disease-free survival (88\% vs 77\%; HR $=0.50 ; 95 \% \mathrm{CI}, 0.39-0.64 ; \mathrm{P}<0.001)$ and the distant recurrence as the first invasive-disease event $(10.5 \%$ vs $15.9 \%) .{ }^{36}$ For those patients that achieve pCR following HER2-directed therapy, adjuvant trastuzumab is continued with or without pertuzumab to complete twelve months of HER2-directed therapy (Figure 1).

The benefit of adding trastuzumab to the adjuvant chemotherapy regimen has been proven in the BCIRG 006 study which demonstrated that both doxorubicincyclophosphamide-docetaxel plus trastuzumab (AC-T plus trastuzumab) and docetaxel-carboplatin-trastuzumab (TCH) were superior to AC-T with respect to DFS (DFS at 5 years; $84 \%$ AC-T plus trastuzumab vs $81 \%$ TCH vs $75 \%$ AC-T). Estimated OS was $92 \%, 91 \%$, and $87 \%$, respectively. ${ }^{37}$ The APHINITY trial demonstrated an improvement in 3-year IDFS with the addition of pertuzumab to adjuvant trastuzumab $(94.1 \%$ with pertuzumab vs 93.2\% with placebo, HR 0.81, 95\% CI 0.66-1.00; $\mathrm{p}=0.045) .{ }^{38}$ Among patients with LN-positive breast cancer, those randomized to receive adjuvant dual HER2 blockade for 1 year following chemotherapy had improved 3 -year IDFS by $1.8 \%$ (92 vs $90.2 \%$, HR $0.77,95 \% \mathrm{CI}$ $0.62-0.96 ; \mathrm{p}=0.02)$. In the cohort with node-negative disease, the 3-year rate of invasive disease-free survival (IDFS) $\backslash$ was $97.5 \%$ in the pertuzumab group and $98.4 \%$ in the placebo group (HR1.13; 95\% CI 0.68-1.86; $\mathrm{P}=0.64)$. These results suggest that in the adjuvant treatment of HER2-positive patients, dual HER2 blockade with

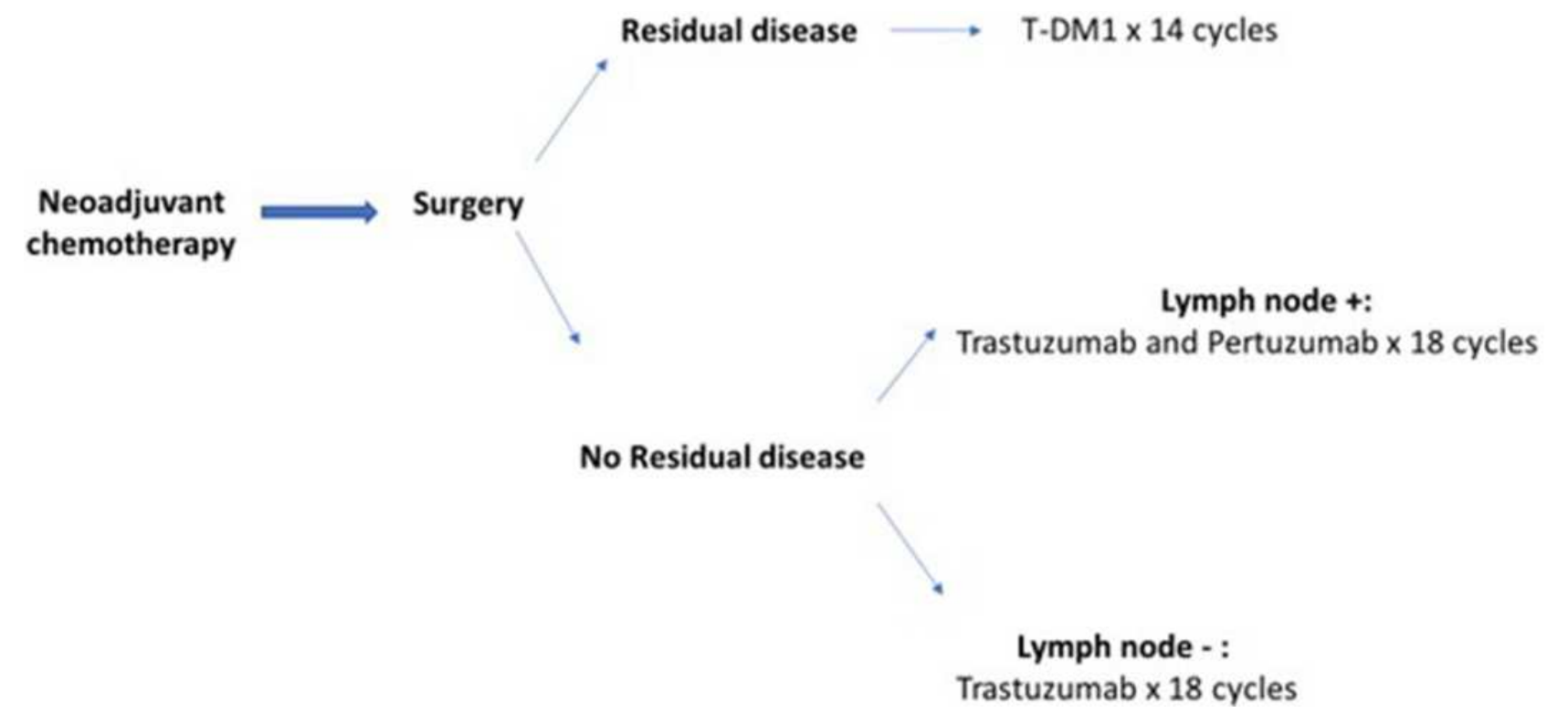

Figure I Treatment algorithm for adjuvant treatment in HER2+ patients. 
trastuzumab plus pertuzumab is valid options for nodepositive disease.

The NCCN Guidelines suggest all HER2-positive breast cancer should be treated with a combination of adjuvant chemotherapy with trastuzumab. ${ }^{39}$ The majority of adjuvant trastuzumab studies incorporated anthracyclines with or without taxanes. The decision on the type of chemotherapy backbone should be tailored based on the risk-benefit ratio for the individual patient. For example, in the aforementioned BCIRG 006 trial, although TCH and AC-T plus trastuzumab had similar DFS and OS, the former showed a more favorable cardiac safety profile. ${ }^{36}$ This chemotherapy regimen would be more suitable for patients with cardiac risk factors such as hypertension, decreased left ventricular ejection fraction, and older age. For HER2-positive tumors $<2 \mathrm{~cm}\left(\mathrm{~T} 1_{\mathrm{a}, \mathrm{b}} \mathrm{N} 0 \mathrm{M} 0\right)$, retrospective studies not only demonstrate a benefit for the administration of trastuzumab but also suggest that there is a significant risk of relapse and adverse prognosis if treated with chemotherapy alone. ${ }^{40-43}$ Therefore, the NCCN guidelines do recommend that these patients receive trastuzumab in combination with chemotherapy.

\section{High-Risk Triple-Negative Breast Cancer}

Triple-negative breast cancer (TNBC) accounts for $15 \%$ of all breast cancer and is characterized by the lack of expression of ER, PR, and HER2. This subtype of breast cancer tends to be very aggressive with early recurrence and worse OS when compared to other breast cancer subtypes. $^{44}$ Neoadjuvant chemotherapy is a standard approach for any tumor greater than $2 \mathrm{~cm}$ or with lymph node involvement. About 35 to $40 \%$ of TNBC patients who receive NACT have a pCR which is a surrogate marker for survival. ${ }^{45}$ However, patients who do not achieve a pCR have poorer outcomes. ${ }^{46}$ At the 2018 SABCS meeting, a meta-analysis of 52 studies, including over 27,000 patients, demonstrated significantly superior 5-year EFS and OS in patients with pCR compared with residual disease $(\mathrm{RD})$, with the greatest difference in the TNBC cohort (90\% vs 57\%). ${ }^{47}$

Early TNBC with tumor sizes $\leq 0.5 \mathrm{~cm}$ (T1a) or between $0.6-1.0 \mathrm{~cm}$ (T1b) without lymph node involvement have a good prognosis without the addition of chemotherapy. A study involving 363 patients with $\mathrm{T}_{\mathrm{a}, \mathrm{b}} \mathrm{N} 0$ TNBC measured the five-year distant recurrence-free survival (DRFS) between patients treated with adjuvant chemotherapy to those who were not. Patients with untreated T1a and T1b TNBC had a five-year DRFS 93\% and 90\% compared to treated T1a and T1b TNBC DRFS $100 \%$ and $96 \%$, respectively. ${ }^{48}$ Therefore, the decision to administer chemotherapy can be made on a case-by-case basis. However, for tumor size $>1 \mathrm{~cm}$ with or without lymph node involvement, systemic chemotherapy is the recommended backbone of treatment. Standard chemotherapy regimens for TNBC are a combination of anthracyclines, taxanes, and alkylators. The Anthracycline in Breast Cancer (ABC) analysis evaluated anthracycline-based regimens versus six cycles of TC in the adjuvant setting and found that overall, patients treated with anthracycline did slightly better (four-year IDFS of $91 \%$ vs $88 \%$ for TC). ${ }^{49}$ The benefit of adding the anthracycline was substantial only in node-positive TNBC and HRpositive patients with four or more involved nodes. Although the ABC trial was performed in the adjuvant setting, the results are commonly extrapolated for management in the neoadjuvant setting.

Platinum agents such as carboplatin have been investigated in the neoadjuvant setting and have consistently been shown to improve $\mathrm{pCR}$ rates in multiple trials including CALGB 40,603, GeparSixto, and BrighTNess (Table 2). In the CALGB 40,603 study, TNBC patients were randomized to receive bevacizumab, carboplatin, or both in combination with standard taxane/anthracycline-based NACT. ${ }^{50}$ Although the addition of carboplatin to standard NACT increased rates of

Table 2 Neoadjuvant Trials in Triple-Negative Patients

\begin{tabular}{|c|c|c|c|c|c|}
\hline \multicolumn{6}{|c|}{ Neoadjuvant Regimens with Carboplatin for Triple-Negative Breast Cancer } \\
\hline Trial & Phase & \# of Patients & Investigational Arm & PCR (\%) with Platinum vs without & EFS Improved \\
\hline CALGB 40,603 & 2 & 443 & $\mathrm{~T} \rightarrow \mathrm{AC}+\mathrm{Bev}$ & 54 vs $4 I \quad(p=0.003)$ & No \\
\hline BrighTNess & 3 & 634 & $\mathrm{~T} \pm \mathrm{V} \rightarrow \mathrm{AC}$ & 58 vs $31(p<0.001)$ & NA \\
\hline GeparSixto & 2 & 595 & $T+A+B e v$ & 53 vs $43(p=0.015)$ & Yes \\
\hline GEICAM/2006-03 & 2 & 189 & $\mathrm{EC} \rightarrow \mathrm{T}+\mathrm{Cb}$ & 30 in both arms & NA \\
\hline
\end{tabular}

Abbreviations: A, anthracycline; Bev, bevacizumab; C, cyclophosphamide; Cb, carboplatin; E, epirubicin; Nab-pac, nabpaclitaxel; pCR, pathologic complete response; T, taxane; V, veliparib. 
pCR from $39 \%$ to $49 \%$, it did not result in improved 5-year EFS and OS. In contrast, the GeparSixto trial demonstrated that the addition of carboplatin significantly improved pCR (53\% vs $43 \%, \mathrm{p}=0.005)$ which resulted in higher rates of 3 -year EFS ( $86 \%$ vs $76 \%$, HR of $0.60,95 \%$ CI $0.34-0.93) .{ }^{51}$ In the BrighTNess study, patients with stage II to III TNBC were randomized to weekly paclitaxel with or without carboplatin and veliparib (an oral PARP inhibitor) followed by doxorubicin and cyclophosphamide. The addition of carboplatin improved the $\mathrm{pCR}$ rate from $31 \%$ to $58 \%$ while the addition of veliparib had no effect on pCR rates. ${ }^{52}$ Long-term outcome data of the BrighTNess trial are still pending.

Interestingly, these studies also demonstrate that TNBC patients are likely to derive benefit from the addition of carboplatin regardless of BRCA status. In BrighTNess, the addition of carboplatin increased the pCR rate in BRCAwildtype patients from 29 to $59 \%$, versus an increase from 41 to $50 \%$ in BRCA carriers. $^{51}$ Additionally, in GeparSixto, there was an absolute improvement in DFS at 35 months with the addition of carboplatin, which was greater in BRCA-wildtype patients (85 versus 74\%; HR 0.53 ; 95\% CI 0.29-0.96), versus in BRCA-mutated patients (86 versus $82 \%) .{ }^{53,54}$ Although the addition of platinum to a NACT regimen leads to higher $\mathrm{pCR}$ rates, the associated toxicities often necessitating dose reductions or cycle eliminations and the contradictory longterm survival results make it a controversial choice.

The recent approval of immune-checkpoint inhibitors (ICI) combined with chemotherapy in programmed deathligand 1 (PD-L1)-positive metastatic TNBC patients has prompted interest in their role in the neoadjuvant setting. ${ }^{55}$ Three clinical trials have shown promising data about the combination of immunotherapy with NACT (Table 3). In Investigation of Serial Studies to Predict Your Therapeutic Response through Imaging and Molecular Analysis 2 (I-SPY2), pembrolizumab (a PD-1 inhibitor) was added to standard NACT to evaluate its success in a confirmatory randomized phase II trial. ${ }^{56}$ Pembrolizumab tripled the estimated pCR rates in TNBC $(60 \%$ with pembrolizumab versus $22 \%$ with placebo). The Phase III KEYNOTE-522 study examined the addition of pembrolizumab to NACT, consisting of paclitaxel and carboplatin followed by doxorubicin or epirubicin and cyclophosphamide, resulted in an improvement in overall pCR rate from 51 to $65 \%$ $(\mathrm{p}<0.001){ }^{57}$ However, additional data about EFS are pending final analysis. The phase III IMpassion031 trial examined the addition of atezolizumab (a PD-L1 inhibitor) to NACT with weekly nab-paclitaxel followed by doxorubicin and cyclophosphamide which resulted in improved pCR rate from 41 to $58 \%(\mathrm{p}=0.0044) .{ }^{58}$ Unfortunately, this study was not powered to address EFS. Both KEYNOTE522 and IMpassion031 showed improved pCR in the PDL1 positive groups, but there was a treatment effect in both the PD-L1 positive and negative groups. Interestingly, the NeoTRIPaPDL1 study did not demonstrate statistically significant improved pCR rates with the addition of atezolizumab to NACT consisting of nab-paclitaxel and carboplatin. $^{59}$

There has been interest in identifying subsets of TNBC patients who may benefit from less chemotherapy due to increased sensitivity to specific agents. One subset of patients who may respond to targeted therapy and require less chemotherapy is those with germline BRCA mutations that are sensitive to poly ADP-ribose polymerases (PARP) inhibitors. Olaparib and talazoparib are two PARP inhibitors currently approved for patients with metastatic breast cancer and germline mutations. A phase II pilot study involved 20 patients (10 TNBC) with germline BRCA mutations receiving neoadjuvant talazoparib in which pCR was achieved in 53\% prompting the exploration of the role of PARP monotherapy in this subset of patients.

Table 3 Neoadjuvant Trials with Immunotherapy in Triple-Negative Patients

\begin{tabular}{|l|l|l|l|l|l|}
\hline \multicolumn{2}{|l|}{ Neoadjuvant Regimens with Immunotherapy in Triple-Negative Breast Cancer } \\
\hline Trial & Phase & $\begin{array}{l}\text { \# of } \\
\text { Patients }\end{array}$ & $\begin{array}{l}\text { Chemotherapy } \\
\text { Regimen }\end{array}$ & $\begin{array}{l}\text { Immune Checkpoint } \\
\text { Inhibitor }\end{array}$ & $\begin{array}{l}\text { pCR(\%) with ICI Versus } \\
\text { without }\end{array}$ \\
\hline I-SPY2 & 2 & 250 & T $\rightarrow$ AC & Pembrolizumab & $60 \%$ vs $22 \%$ \\
KEYNOTE-522 & 3 & 602 & Cb + T $\rightarrow$ AC & Pembrolizumab & $65 \%$ vs $51 \%(p<0.00$ I) \\
IMpassion03I & 3 & 333 & Nab-pac $\rightarrow$ AC & Atezolizumab & $58 \%$ vs $41 \%(p=0.004)$ \\
NeoTRIPaPDLI & 3 & 280 & Cb + Nab-pac & Atezolizumab & $44 \%$ vs $41 \%(p=0.66)$ \\
\hline
\end{tabular}

Abbreviations: A, anthracycline; Bev, bevacizumab; C, cyclophosphamide; Cb, carboplatin; Nab-pac, nabpaclitaxel; pCR, pathologic complete response; T, taxane; $\mathrm{V}$, veliparib. 
In the case of residual disease after NACT, adjuvant capecitabine is another potential therapy to further reduce the risk of breast cancer recurrence. The CREATE-X trial randomized 910 patients with HER2-negative disease treated with standard NACT and with residual disease to adjuvant capecitabine for six to eight cycles versus observation only. ${ }^{60}$ Adjuvant radiotherapy was given if indicated. Full analysis demonstrated an improvement in 5 -year DFS from $69.8 \%$ to $56.1 \%$ with capecitabine in the TNBC subset. These findings further support the use of NACT instead of adjuvant chemotherapy in TNBC patients, as those patients who achieve $\mathrm{pCR}$ have improved outcomes and those that do not achieve pCR clearly can benefit from the addition of adjuvant capecitabine.

\section{Inflammatory Breast Cancer}

Inflammatory breast cancer (IBC) is a rare form of breast cancer comprising $2.5 \%$ of all breast cancers in the United States. ${ }^{61}$ The main role of neoadjuvant therapy in the setting of IBC is to be able to surgically treat with modified radical mastectomy without leaving positive margins, thereby limiting the risk of systemic metastases.

The current standard of neoadjuvant therapy in IBC is determined by the subtype of breast cancer with regard to expression of ER, PR, and HER2. Neoadjuvant therapy for ER-positive and TNBC remains anthracycline and taxane-based chemotherapy. In a large retrospective study, of the 178 IBC patients treated with anthracyclinebased chemotherapy followed by local treatment with irradiation, with or without mastectomy, 15-year survival was the greatest in patients who accomplished pCR (44\%), versus partial response (31\%). ${ }^{62}$ In a cohort study of 68 patients with IBC, treatment with either NACT consisting of FEC (cyclophosphamide, epirubicin and 5-fluorouracil) or FAC (cyclophosphamide, doxorubicin and 5-fluorouracil), followed by surgery, adjuvant therapy, and radiotherapy resulted in overall 5-year survival rate of $44 \%$ and 10 -year survival of $32 \% .{ }^{63}$ These results confirmed that IBC patients obtained significant long-term survival benefit from combined-modality treatment. The standard of care in neoadjuvant treatment of IBC is a sequential regimen using anthracyclines then taxanes.

Given that IBC has a higher proportion of HER2positive tumors as compared to noninflammatory cancers or other locally advanced cancers, anti-HER 2 treatments have an integral role in the neoadjuvant setting. In the
NeOAdjuvant Herceptin (NOAH) trial, HER2-positive IBC had a pCR of $54.8 \%$ after treatment with neoadjuvant trastuzumab combined with chemotherapy. ${ }^{64} \mathrm{~A}$ subsequent subgroup analysis comparing patients that received a combination of trastuzumab plus NACT vs NACT alone had a better 5 -year event-free survival (64\% vs $24 \%)$ and 5 -year OS $(74 \%$ vs $44 \%))^{33}$

\section{Locally Advanced Breast Cancer or Clinically Node-Positive Disease}

Patients with $\mathrm{T} 3$ or $\mathrm{T} 4$ breast lesions or with multiple axillary lymph nodes involved are candidates for NACT as they are often not amenable to upfront resection and they would not be candidates for breast conservation. Additionally, patients with limited clinically nodepositive disease are also eligible for NACT, as it often converts $\mathrm{cN} 1$ patients to $\mathrm{pN} 0$, especially in patients with aggressive subtypes.

Neoadjuvant therapy was initially pursued in patients with locally advanced breast cancers, typically at least $\mathrm{T} 3(\geq 5 \mathrm{~cm}$ in largest dimension), in which only larger, involved surgeries would have been considered. Patients with locally advanced breast cancers or inoperable breast cancers have traditionally been excluded from larger randomized control trials assessing the effect of neoadjuvant therapy on surgical options and outcomes. However, neoadjuvant therapy in this group of patients is recommended by major cancer society governing bodies $\mathrm{ASCO}^{65}$ and NCCN. ${ }^{39}$ The rationale is that neoadjuvant systemic therapy can lead to downstaging of the primary tumor to increase operability or potential cosmesis of breastconserving therapy (BCT), as well as to more rapidly treat subclinical distant micrometastases. However, there have been several large randomized controlled trials that have clearly demonstrated improved rates of BCT therapy with neoadjuvant therapy compared to adjuvant therapy in earlier stage operable breast cancers. This data has been extended to guide management of more locally advanced disease. NSABP B-18 randomized 1523 women with stage III disease to either pre-operative or post-operative AC. ${ }^{66}$ It found that more patients receiving neoadjuvant therapy were able to receive lumpectomy, compared to those receiving adjuvant therapy $(67.8 \%$ vs $59.8 \%)$. The ECTO trial assessed patients with primary operable breast cancer $\left(\mathrm{T}_{2-3} \mathrm{~N}_{0-1} \mathrm{M}_{0}\right)$ who received neoadjuvant systemic therapy (in this case AT x 4 cycles, followed by CMF for 4 cycles) and compared them to those receiving surgery followed by adjuvant therapy. ${ }^{67}$ There was a significant reduction in the 
requirement for mastectomy in patients who received neoadjuvant therapy. $65 \%$ of patients receiving neoadjuvant therapy were able to receive BCS compared to $34 \%$ of women in the primary surgery arms $(\mathrm{p}<0.001)$.

More importantly, several studies have performed secondary analyses which demonstrated that patients who were not candidates for breast-conserving surgery at diagnosis were able to become candidates for BCS after neoadjuvant therapy. In the CALGB 40,601 study, breast-conserving surgery candidacy was assessed of patients with stage II-III HER2+ breast cancer before and after neoadjuvant therapy. ${ }^{68} 43 \%$ of the patients who were not initially candidates for BCS converted to candidates for BCS after neoadjuvant therapy. In the more recent BrighTNess RCT, 604 patients with stage II-III TNBC were assessed for BCS candidacy before and after neoadjuvant therapy. ${ }^{69}$ Of the 141 patients who were deemed BCS ineligible at baseline, 53.2\% then converted to BCS eligible after neoadjuvant therapy with $\mathrm{ACT} \pm$ carboplatin and/or veliparib.

Traditionally, the standard approach to clinically nodepositive breast cancer is with axillary lymph node dissection (ALND). However, ALND has been associated with higher surgical complications and long-term symptomatic risks of lymphedema, limited mobility in that extremity, and neuropathy which can greatly lessen quality of life. Currently, randomized controlled trials examining the question of whether neoadjuvant therapy can decrease the need for ALND are ongoing. However, guidelines still recommend upfront systemic therapy for clinically nodepositive disease to permit for less extensive surgery on the axilla based on data from other trials. ${ }^{1}$ In a prospective study of 288 node-positive stage II-III breast cancer patients receiving neoadjuvant therapy, patients who were clinically node-negative after treatment were candidates for sentinel lymph node biopsy (SLNB). ${ }^{70} 68 \%$ of those who underwent surgery became clinically node-negative after NACT and were able to undergo an SLNB as opposed to ALND. Of the 128 SLNB cases, 48\% were able to avoid a subsequent ALND, which supports the role of neoadjuvant therapy for reducing the need for ALND among patients with metastases. One significant concern with neoadjuvant therapy followed by SLNB instead of ALND in patients who were baseline clinically nodepositive is the potential false-negative rate of SLNB; i.e. no evidence of nodal metastases on sufficient SLNB, but later found to have nodal metastases on an ALND. The ACOSOG Z1071 trial specifically addressed this concern. The initial multi-institutional trial demonstrated that the false-negative rate of SLNB after NACT was $12.6 \%{ }^{71}$ However, a secondary analysis of the study showed that in patients who had a clip placed in the positive node at the initial biopsy and had an SLNB of at least 2 nodes, that the clip was recovered within the SLN specimen $83.7 \%$ of the time, ${ }^{72}$ Another study showed that in 118 patients undergoing SLNB followed by ALND, that if the clipped node was included in the SLNB that the false-negative rate decreased greatly, from $10.1 \%$ to $1.4 \%{ }^{73}$ Thus, a reasonable approach to clinically nodepositive disease is to place a clip during biopsy, pursue neoadjuvant therapy, and then if then downstaged to clinically node-negative disease move forward with an SLNB. SLNB should include the clip to decrease the false-negative rate. If SLNB is positive for nodal metastasis, then ALND can be performed. If SLNB is negative, potential complications and sequelae of ALND can be avoided.

\section{Patients with Contraindications for Surgery}

Neoadjuvant chemotherapy or endocrine therapy may be a suitable option for patients who have contraindications to undergo surgery at the time of diagnosis or in situations in which delays in elective surgeries are encountered or necessitated, such as during a pandemic, during pregnancy or after a recent venous thromboembolism.

There are several absolute contraindications to upfront surgery and/or radiation. These typically are due to inability to achieve appropriate margin control with BCS or inability of the patient to tolerate radiation therapy. Some of the absolute contraindications to initial BCS are multicentric disease where tumors are located in different quadrants of the breast and patients with diffuse malignant-appearing microcalcifications not confined to a single localized area. Due to the multicentric or diffuse nature of these presentations, a lumpectomy or smaller surgery is often not able to be completed until downstaging by neoadjuvant therapy. Patients who have had prior radiation therapy to the affected breast also have an absolute contraindication to BCT. Frequently, these patients have had previous radiation in the setting of treatment of Hodgkin's Lymphoma or previous breast cancer. If they were considered for further radiation, these patients would receive unacceptably high doses of radiation, making BCT infeasible. An additional absolute contraindication to $\mathrm{BCT}$ is a patient diagnosed in the first or second 
trimester of pregnancy and where a pregnancy termination is not desired or feasible. This is because therapeutic radiation during pregnancy is teratogenic to the fetus. Typically, radiation is given within 3-8 weeks of lumpectomy. Waiting a longer period of time, such as greater than 20 weeks, can significantly decrease local recurrence-free survival and breast cancer-specific survival, as compared to waiting $4-8$ weeks after therapy. ${ }^{74}$ In this situation, it is reasonable to consider neoadjuvant therapy. For patients diagnosed in the third trimester, they may be able to move forward with upfront BCT as radiation therapy may be administered after delivery of the baby.

Some relative contraindications to initial BCT are those that are related to vascular changes of the patient, which increase the risk of complications or the risk of inadequate cosmesis of the breast. Traditionally, patients with collagen vascular disease (CVD) have been classified as having a relative contraindication to $\mathrm{BCT}$ due to poor vasculature and cutaneous friability, making radiation complications more likely. However, there have been multiple studies that have shown that the increased risk of radiation complications of CVD patients is only in scleroderma patients. ${ }^{75,76}$ This is not seen in other patients with CVD. Another relative contraindication to initial surgery is an insufficient tumor-to-breast ratio (i.e. a large tumor in a small breast), which would not allow for acceptable cosmetic result. ${ }^{77}$ In these cases, neoadjuvant systemic therapy can allow for downstaging of the tumor and allow for a more amenable tumor to breast size ratio and improved cosmetic outcome. Other relative contraindications to primary surgery and radiation may be additional medical or social issues; such as recent thromboembolism requiring anticoagulation, temporary medical unfitness, or delay of non-urgent procedures given medical system strain during a pandemic. In these cases, NACT or NET - if clinically appropriate - can help to manage and treat the breast cancer until the patient is able to receive surgery and radiation.

\section{Conclusion}

The landscape of neoadjuvant therapy continues to evolve. Advances in systemic therapies administered in the neoadjuvant setting have resulted in increased suitability for subsequent surgeries. Clinical trials continue to investigate other therapies (ICI, PI3K inhibitors, mTOR inhibitors) that can be utilized for the different subtypes of breast cancer. The I-SPY2 provides a unique opportunity to evaluate novel agents added to standard neoadjuvant therapy in small study populations with an earlier endpoint of pCR. This allows investigational drugs with a high probability of efficacy to move on to phase III studies more rapidly. ${ }^{78}$ Another area of interest is developing biomarkers that can be predictors and prognosticators of response to neoadjuvant treatment. Biomarkers (TIL, tumor PD-L1, germline BRCA) can identify patients likely to achieve pCR, predict who can forgo surgery, and detect minimal residual disease (ctDNA and circulating tumor cells). Understanding the clinical, genetic, and molecular profiles in breast cancer leads to the development of validated predictive and prognostic tools that helps individualize treatment plans for these patients.

\section{Author Contributions}

All authors made a significant contribution to the work reported, whether that is in the conception, study design, execution, acquisition of data, analysis and interpretation, or in all these areas; took part in drafting, revising or critically reviewing the article; gave final approval of the version to be published; have agreed on the journal to which the article has been submitted; and agree to be accountable for all aspects of the work.

\section{Disclosure}

All authors declared that there are no conflicts of interest in this work.

\section{References}

1. DeSantis CE, Ma J, Gaudet MM, et al. Breast cancer statistics, 2019. CA Cancer J Clin. 2019;69(6):438-451. doi:10.3322/caac.21583

2. Asselain B, Barlow W, Bartlett J, Early Breast Cancer Trialists' Collaborative Group (EBCTCG). Long-term outcomes for neoadjuvant versus adjuvant chemotherapy in early breast cancer: meta-analysis of individual patient data from ten randomised trials. Lancet Oncol. 2018;19(1):27-39. doi:10.1016/S1470-2045(17)307775

3. Wolmark N, Wang J, Mamounas E, Bryant J, Fisher B. Preoperative chemotherapy in patients with operable breast cancer: nine-year results from National Surgical Adjuvant Breast and Bowel Project B-18. J Natl Cancer Inst Monogr. 2001;2001(30):96-102. doi:10.1093/ oxfordjournals.jncimonographs.a003469

4. Spring LM, Fell G, Arfe A, et al. Pathologic complete response after neoadjuvant chemotherapy and impact on breast cancer recurrence and survival: a comprehensive meta-analysis. Clin Cancer Res. 2020;26 (12):2838-2848. doi:10.1158/1078-0432.CCR-19-3492

5. Cortazar P, Geyer CE Jr. Pathological complete response in neoadjuvant treatment of breast cancer. Ann Surg Oncol. 2015;22 (5):1441-1446. doi:10.1245/s10434-015-4404-8

6. Cortazar P, Zhang L, Untch M, et al. Pathological complete response and long-term clinical benefit in breast cancer: the CTNeoBC pooled analysis [published correction appears in Lancet. 2019 Mar 9;393(10175):986]. Lancet. 2014;384(9938):164-172. doi:10.1016/ S0140-6736(13)62422-8 
7. Kuerer HM, Newman LA, Smith TL, et al. Clinical course of breast cancer patients with complete pathologic primary tumor and axillary lymph node response to doxorubicin-based neoadjuvant chemotherapy. J Clin Oncol. 1999;17(2):460-469. doi:10.1200/ JCO.1999.17.2.460

8. Guarneri V, Broglio K, Kau SW, et al. Prognostic value of pathological complete response after primary chemotherapy in relation to hormone receptor status and other factors. $J$ Clin Oncol. 2006;24:1037-1044. doi:10.1200/JCO.2005.02.6914

9. Howlader N, Altekruse SF, Li CI, et al. US incidence of breast cancer subtypes defined by joint hormone receptor and HER2 status. J Natl Cancer Inst. 2014;106(5):dju055. doi:10.1093/jnci/dju055

10. Spring LM, Gupta A, Reynolds KL, et al. Neoadjuvant endocrine therapy for estrogen receptor-positive breast cancer: a systematic review and meta-analysis. JAMA Oncol. 2016;2(11):1477-1486. doi:10.1001/jamaoncol.2016.1897

11. Eiermann W, Paepke S, Appfelstaedt J, et al. Preoperative treatment of postmenopausal breast cancer patients with letrozole: a randomized double-blind multicenter study. Ann Oncol. 2001;12 (11):1527-1532. doi:10.1023/a:1013128213451

12. Cataliotti L, Buzdar AU, Noguchi S, et al. Comparison of anastrozole versus tamoxifen as preoperative therapy in postmenopausal women with hormone receptor-positive breast cancer: the Pre-Operative "Arimidex" Compared to Tamoxifen (PROACT) trial. Cancer. 2006;106(10):2095-2103. doi:10.1002/cncr.21872

13. Smith IE, Dowsett M, Ebbs SR, et al. Neoadjuvant treatment of postmenopausal breast cancer with anastrozole, tamoxifen, or both in combination: the Immediate Preoperative Anastrozole, Tamoxifen, or Combined with Tamoxifen (IMPACT) multicenter double-blind randomized trial. J Clin Oncol. 2005;23(22):5108-5116. doi:10.1200/ JCO.2005.04.005

14. Ellis MJ, Suman VJ, Hoog J, et al. Randomized phase II neoadjuvant comparison between letrozole, anastrozole, and exemestane for postmenopausal women with estrogen receptor-rich stage 2 to 3 breas cancer: clinical and biomarker outcomes and predictive value of the baseline PAM50-based intrinsic subtype--ACOSOG Z1031. J Clin Oncol. 2011;29(17):2342-2349. doi:10.1200/JCO.2010.31.6950

15. Carpenter R, Doughty JC, Cordiner C, et al. Optimum duration of neoadjuvant letrozole to permit breast conserving surgery. Breast Cancer Res Treat. 2014;144(3):569-576. doi:10.1007/s10549-0142835-8

16. Krainick-Strobel UE, Lichtenegger W, Wallwiener D, et al. Neoadjuvant letrozole in postmenopausal estrogen and/or progesterone receptor positive breast cancer: a phase IIb/III trial to investigate optimal duration of preoperative endocrine therapy. BMC Cancer. 2008;8:62. doi:10.1186/1471-2407-8-62

17. Rusz O, Vörös A, Varga Z, et al. One-year neoadjuvant endocrine therapy in breast cancer. Pathol Oncol Res. 2015;21(4):977-984. doi:10.1007/s12253-015-9911-1

18. Fontein DB, Charehbili A, Nortier JW, et al. Efficacy of six month neoadjuvant endocrine therapy in postmenopausal, hormone receptor-positive breast cancer patients--a phase II trial. Eur J Cancer. 2014;50(13):2190-2200. doi:10.1016/j.ejca.2014.05.010

19. Ellis MJ, Tao Y, Luo J, et al. Outcome prediction for estrogen receptor-positive breast cancer based on postneoadjuvant endocrine therapy tumor characteristics. J Natl Cancer Inst. 2008;100 (19):1380-1388. doi:10.1093/jnci/djn309

20. Ellis MJ, Suman VJ, Hoog J, et al. Ki67 proliferation index as a tool for chemotherapy decisions during and after neoadjuvant aromatase inhibitor treatment of breast cancer: results from the American College of Surgeons Oncology Group Z1031 trial (alliance). J Clin Oncol. 2017;35(10):1061-1069. doi:10.1200/JCO.2016.69.4406

21. Suman VJ, Ellis MJ, Ma CX. The ALTERNATE trial: assessing a biomarker driven strategy for the treatment of post-menopausal women with ER+/Her2- invasive breast cancer. Chin Clin Oncol. 2015;4(3):34. doi:10.3978/j.issn.2304-3865.2015.09.01
22. Hurvitz SA, Martin M, Press MF, et al. Potent cell-cycle inhibition and upregulation of immune response with abemaciclib and anastrozole in neoMONARCH, phase II Neoadjuvant Study in HR+/HER2- breast cancer. Clin Cancer Res. 2020;26(3):566-580. doi:10.1158/1078-0432. CCR-19-1425

23. Johnston S, Puhalla S, Wheatley D, et al. Randomized Phase II Study evaluating palbociclib in addition to letrozole as neoadjuvant therapy in estrogen receptor-positive early breast cancer: PALLET trial. J Clin Oncol. 2019;37(3):178-189. doi:10.1200/JCO.18.01624

24. Prat A, Saura C, Pascual T, et al. Ribociclib plus letrozole versus chemotherapy for postmenopausal women with hormone receptor-positive, HER2-negative, luminal $B$ breast cancer (CORALLEEN): an open-label, multicentre, randomised, phase 2 trial. Lancet Oncol. 2020;21(1):33-43. doi:10.1016/S1470-2045(19)30786-7

25. Saura C, Hlauschek D, Oliveira M, et al. Neoadjuvant letrozole plus taselisib versus letrozole plus placebo in postmenopausal women with oestrogen receptor-positive, HER2-negative, early-stage breast cancer (LORELEI): a multicentre, randomised, double-blind, placebo-controlled, phase 2 trial. Lancet Oncol. 2019;20 (9):1226-1238. doi:10.1016/S1470-2045(19)30334-1

26. Loi S, McArthur HL, Harbeck N, et al. A phase III trial of nivolumab with neoadjuvant chemotherapy and adjuvant endocrine therapy in ER+/HER2- primary breast cancer: checkMate 7FL. J Clin Oncol. 2020;38(15_suppl):TPS604-TPS604. doi:10.1200/ JCO.2020.38.15_suppl.TPS604

27. Cardoso F, Bardia A, Andre F, et al. KEYNOTE-756: a randomized, double-blind, phase III study of pembrolizumab versus placebo in combination with neoadjuvant chemotherapy and adjuvant endocrine therapy for high-risk early-stage ER+/HER2- breast cancer [abstract] In: Proceedings of the 2018 San Antonio Breast Cancer Symposium; Dec 4-8, 2018; San Antonio, TX. Philadelphia (PA): AACR. Cancer Res 2019; 79(4Suppl): Abstract nr OT3-04-03. doi: 10.1158/1538-7445.SABCS18-OT3-04-03

28. Slamon DJ, Clark GM, Wong SG, Levin WJ, Ullrich A, McGuire WL. Human breast cancer: correlation of relapse and survival with amplification of the HER-2/neu oncogene. Science. 1987;235(4785):177-182. doi:10.1126/science.3798106

29. Broglio KR, Quintana M, Foster M, et al. Association of pathologic complete response to neoadjuvant therapy in HER2-positive breast cancer with long-term outcomes: a meta-analysis. JAMA Oncol. 2016;2(6):751-760. doi:10.1001/jamaoncol.2015.6113

30. Robidoux A, Tang G, Rastogi P, et al. Lapatinib as a component of neoadjuvant therapy for HER2-positive operable breast cancer (NSABP protocol B-41): an open-label, randomised phase 3 trial. Lancet Oncol. 2013;14(12):1183-1192. doi:10.1016/S1470-2045(13)70411-X

31. van der Voort A, van Ramshorst MS, van Werkhoven ED, et al. Three-year follow-up of neoadjuvant chemotherapy with or without anthracyclines in the presence of dual HER2-blockade for HER2-positive breast cancer (TRAIN-2): a randomized phase III trial. J Clin Oncol. 2020;38S:ASCO \#501.

32. Schneeweiss A, Chia S, Hickish T, et al. Pertuzumab plus trastuzu$\mathrm{mab}$ in combination with standard neoadjuvant anthracycline-containing and anthracycline-free chemotherapy regimens in patients with HER2-positive early breast cancer: a randomized phase II cardiac safety study (TRYPHAENA). Ann Oncol. 2013;24(9):2278-2284. doi:10.1093/annonc/mdt182

33. Gianni L, Eiermann W, Semiglazov V, et al. Neoadjuvant and adjuvant trastuzumab in patients with HER2-positive locally advanced breast cancer (NOAH): follow-up of a randomised controlled superiority trial with a parallel HER2-negative cohort [published correction appears in Lancet Oncol. 2018 Dec;19(12):e667]. Lancet Oncol. 2014;15(6):640-647. doi:10.1016/S1470-2045(14)70080-4

34. Gianni L, Pienkowski T, Im YH, et al. Efficacy and safety of neoadjuvant pertuzumab and trastuzumab in women with locally advanced, inflammatory, or early HER2-positive breast cancer (NeoSphere): a randomised multicentre, open-label, phase 2 trial. Lancet Oncol. 2012;13(1):25-32. doi:10.1016/S1470-2045(11)70336-9 
35. Bria E, Carbognin L, Furlanetto J, et al. Impact of neoadjuvant single or dual HER2 inhibition and chemotherapy backbone upon pathological complete response in operable and locally advanced breast cancer: sensitivity analysis of randomized trials. Cancer Treat Rev. 2014;40(7):847-856. doi:10.1016/j.ctrv.2014.05.001

36. von Minckwitz G, Huang CS, Mano MS, et al. Trastuzumab emtansine for residual invasive HER2-positive breast cancer. $N$ Engl J Med. 2019;380(7):617-628. doi:10.1056/NEJMoa1814017

37. Slamon D, Eiermann W, Robert N, et al. Adjuvant trastuzumab in HER2-positive breast cancer. $N$ Engl J Med. 2011;365 (14):1273-1283. doi:10.1056/NEJMoa0910383

38. von Minckwitz G, Procter M, de Azambuja E, et al. Adjuvant pertuzumab and trastuzumab in early HER2-positive breast cancer [published correction appears in N Engl J Med. 2017 Aug 17;377(7):702][published correction appears in N Engl J Med. 2018 Oct 18;379(16):1585]. N Engl J Med. 2017;377(2):122-131. doi:10.1056/NEJMoa1703643

39. National Comprehensive Cancer Network. Breast cancer (Version 1.2021). Available from: https://www.nccn.org/professionals/physi cian_gls/pdf/breast_blocks.pdf. Accessed January 30, 2021.

40. Gonzalez-Angulo AM, Litton JK, Broglio KR, et al. High risk of recurrence for patients with breast cancer who have human epidermal growth factor receptor 2-positive, node-negative tumors $1 \mathrm{~cm}$ or smaller. J Clin Oncol. 2009;27(34):5700-5706. doi:10.1200/ JCO.2009.23.2025

41. Curigliano G, Viale G, Bagnardi V, et al. Clinical relevance of HER2 overexpression/amplification in patients with small tumor size and node-negative breast cancer. J Clin Oncol. 2009;27(34):5693-5699. doi:10.1200/JCO.2009.22.0962

42. Theriault RL, Litton JK, Mittendorf EA, et al. Age and survival estimates in patients who have node-negative T1ab breast cancer by breast cancer subtype. Clin Breast Cancer. 2011;11(5):325-331. doi:10.1016/j.clbc.2011.05.002

43. Joensuu H, Isola J, Lundin M, et al. Amplification of erbB2 and erbB2 expression are superior to estrogen receptor status as risk factors for distant recurrence in pT1N0M0 breast cancer: a nationwide population-based study. Clin Cancer Res. 2003;9 (3):923-930.

44. Howlander N, Noone A, Krapcho M, Miller D, Brest A, Yu M. SEER Cancer Statistics Review, 1975-2017. Bethesda, MD: National Cancer Institute; 2017.

45. Biswas T, Efird JT, Prasad S, Jindal C, Walker PR. The survival benefit of neoadjuvant chemotherapy and pCR among patients with advanced stage triple negative breast cancer. Oncotarget. 2017;8 (68):112712-112719. doi:10.18632/oncotarget.22521

46. von Minckwitz G, Untch M, Blohmer JU, et al. Definition and impact of pathologic complete response on prognosis after neoadjuvant chemotherapy in various intrinsic breast cancer subtypes. J Clin Oncol. 2012;30(15):1796-1804. doi:10.1200/JCO.2011.38.8595

47. Spring LM, Fell G, Arfe A, et al. Pathological complete response (pCR) after neoadjuvant chemotherapy and impact on breast cancer recurrence and survival, stratified by breast cancer subtypes and adjuvant chemotherapy usage: patient-level meta-analyses of over 27,000 patients. Proceedings from the 2018 San Antonio Breast Cancer Symposium (SABCS); December 4-8, 2018; San Antonio, TX.

48. Vaz-Luis I, Ottesen RA, Hughes ME, et al. Outcomes by tumor subtype and treatment pattern in women with small, node-negative breast cancer: a multi-institutional study. J Clin Oncol. 2014;32 (20):2142-2150. doi:10.1200/JCO.2013.53.1608

49. Blum JL, Flynn PJ, Yothers G, et al. Anthracyclines in early breast cancer: the ABC trials-USOR 06-090, NSABP B-46-I/USOR 07132, and NSABP B-49 (NRG oncology). J Clin Oncol. 2017;35 (23):2647-2655. doi:10.1200/JCO.2016.71.4147
50. Sikov WM, Berry DA, Perou CM, et al. Impact of the addition of carboplatin and/or bevacizumab to neoadjuvant once-per-week paclitaxel followed by dose-dense doxorubicin and cyclophosphamide on pathologic complete response rates in stage II to III triple-negative breast cancer: CALGB 40603 (Alliance). J Clin Oncol. 2015;33 (1):13-21. doi:10.1200/JCO.2014.57.0572

51. Yuan Y, Lee JS, Yost SE, et al. Phase II trial of neoadjuvant carboplatin and nab-paclitaxel in patients with triple-negative breast cancer [published online ahead of print, 2020 Oct 24]. Oncologist. 2020. doi:10.1002/onco.13574

52. Loibl S, O'Shaughnessy J, Untch M, et al. Addition of the PARP inhibitor veliparib plus carboplatin or carboplatin alone to standard neoadjuvant chemotherapy in triple-negative breast cancer (BrighTNess): a randomised, phase 3 trial. Lancet Oncol. 2018;19 (4):497-509. doi:10.1016/S1470-2045(18)30111-6

53. Hahnen E, Lederer B, Hauke J, et al. Germline mutation status, pathological complete response, and disease-free survival in triple-negative breast cancer: secondary analysis of the GeparSixto randomized clinical trial. JAMA Oncol. 2017;3(10):1378-1385. doi:10.1001/jamaoncol.2017.1007

54. Loibl S, Weber KE, Timms KM, et al. Survival analysis of carboplatin added to an anthracycline/taxane-based neoadjuvant chemotherapy and HRD score as predictor of response-final results from GeparSixto. Ann Oncol. 2018;29(12):2341-2347. doi:10.1093/ annonc/mdy460

55. Garufi G, Palazzo A, Paris I, et al. Neoadjuvant therapy for triple-negative breast cancer: potential predictive biomarkers of activity and efficacy of platinum chemotherapy, PARP- and immune-checkpoint-inhibitors. Expert Opin Pharmacother. 2020;21 (6):687-699. doi:10.1080/14656566.2020.1724957

56. Nanda R, Liu MC, Yau C, et al. Effect of pembrolizumab plus neoadjuvant chemotherapy on pathologic complete response in women with early-stage breast cancer: an analysis of the ongoing phase 2 adaptively randomized I-SPY2 trial. JAMA Oncol. 2020;6 (5):676-684. doi:10.1001/jamaoncol.2019.6650

57. Schmid P, Cortes J, Pusztai L, et al. Pembrolizumab for early triple-negative breast cancer. $N$ Engl J Med. 2020;382(9):810-821. doi:10.1056/NEJMoa1910549

58. Mittendorf EA, Zhang H, Barrios $\mathrm{CH}$, et al. Neoadjuvant atezolizumab in combination with sequential nab-paclitaxel and anthracycline-based chemotherapy versus placebo and chemotherapy in patients with early-stage triple-negative breast cancer (IMpassion031): a randomised, double-blind, phase 3 trial. Lancet. 2020;396(10257):1090-1100. doi:10.1016/S0140-6736(20)31953-X

59. Gianni L, Huang CS, Egle D, et al. Pathologic complete response (pCR) to neoadjuvant treatment with or without atezolizumab in triple negative, early high-risk and locally advanced breast cancer. NeoTRIPaPDL1 michelangelo randomized study. Cancer Res. 2019;80S:SABCS \#GS3-04.

60. Masuda N, Lee SJ, Ohtani S, et al. Adjuvant capecitabine for breast cancer after preoperative chemotherapy. $N$ Engl J Med. 2017;376 (22):2147-2159. doi:10.1056/NEJMoa1612645

61. Hance KW, Anderson WF, Devesa SS, Young HA, Levine PH. Trends in inflammatory breast carcinoma incidence and survival: the surveillance, epidemiology, and end results program at the National Cancer Institute. J Natl Cancer Inst. 2005;97(13):966-975. doi:10.1093/jnci/dji172

62. Ueno NT, Buzdar AU, Singletary SE, et al. Combined-modality treatment of inflammatory breast carcinoma: twenty years of experience at M. D. Anderson Cancer Center. Cancer Chemother Pharmacol. 1997;40(4):321-329. doi:10.1007/s002800050664

63. Baldini E, Gardin G, Evagelista G, Prochilo T, Collecchi P, Lionetto R. Long-term results of combined-modality therapy for inflammatory breast carcinoma. Clin Breast Cancer. 2004;5 (5):358-363. doi:10.3816/cbc.2004.n.042 
64. Gianni L, Eiermann W, Semiglazov V, et al. Neoadjuvant chemotherapy with trastuzumab followed by adjuvant trastuzumab versus neoadjuvant chemotherapy alone, in patients with HER2-positive locally advanced breast cancer (the NOAH trial): a randomised controlled superiority trial with a parallel HER2-negative cohort. Lancet. 2010;375(9712):377-384. doi:10.1016/S0140-6736(09)61964-4

65. Korde LA, Somerfield MR, Carey LA, et al. Neoadjuvant chemotherapy, endocrine therapy, and targeted therapy for breast Cancer: ASCO guideline [published online ahead of print, 2021 Jan 28]. J Clin Oncol. 2021;JCO2003399. doi:10.1200/JCO.20.03399

66. Fisher B, Bryant J, Wolmark N, et al. Effect of preoperative chemotherapy on the outcome of women with operable breast cancer. J Clin Oncol. 1998;16(8):2672-2685. doi:10.1200/JCO.1998.16.8.2672

67. Gianni L, Baselga J, Eiermann W, et al. Feasibility and tolerability of sequential doxorubicin/paclitaxel followed by cyclophosphamide, methotrexate, and fluorouracil and its effects on tumor response as preoperative therapy. Clin Cancer Res. 2005;11(24 Pt 1):8715-8721. doi:10.1158/1078-0432.CCR-05-0539

68. Golshan M, Cirrincione CT, Sikov WM, et al. Impact of neoadjuvant therapy on eligibility for and frequency of breast conservation in stage II-III HER2-positive breast cancer: surgical results of CALGB 40601 (Alliance). Breast Cancer Res Treat. 2016;160(2):297-304. doi:10.1007/s10549-016-4006-6

69. Golshan M, Loibl S, Wong SM, et al. Breast conservation after neoadjuvant chemotherapy for triple-negative breast cancer: surgical results from the BrighTNess randomized clinical trial. JAMA Surg. 2020;155(3):e195410. doi:10.1001/jamasurg.2019.5410

70. Mamtani A, Av B, Ta K, et al. How often does neoadjuvant chemotherapy avoid axillary dissection in patients with histologically confirmed nodal metastases? Results of a Prospective Study. Ann Surg Oncol. 2016;23(11):3467-3474. doi:10.1245/s10434-016-5246-8

71. Boughey JC, Suman VJ, Mittendorf EA, et al. Sentinel lymph node surgery after neoadjuvant chemotherapy in patients with node-positive breast cancer: the ACOSOG Z1071 (Alliance) clinical trial. JAMA. 2013;310(14):1455-1461. doi:10.1001/jama.2013.278932
72. Boughey JC, Ballman KV, Le-petross HT, et al. Identification and resection of clipped node decreases the false-negative rate of sentinel lymph node surgery in patients presenting with node-positive breast cancer (T0-T4, N1-N2) who receive neoadjuvant chemotherapy: results from ACOSOG Z1071 (alliance). Ann Surg. 2016;263 (4):802-807. doi:10.1097/SLA.0000000000001375

73. Caudle AS, Yang WT, Krishnamurthy S, et al. Improved axillary evaluation following neoadjuvant therapy for patients with node-positive breast cancer using selective evaluation of clipped nodes: implementation of targeted axillary dissection. J Clin Oncol. 2016;34(10):1072-1078. doi:10.1200/JCO.2015.64.0094

74. Olivotto IA, Lesperance ML, Truong PT, et al. Intervals longer than 20 weeks from breast-conserving surgery to radiation therapy are associated with inferior outcome for women with early-stage breast cancer who are not receiving chemotherapy [published correction appears in J Clin Oncol. 2009 May 10;27(14):2415]. J Clin Oncol. 2009;27(1):16-23. doi:10.1200/JCO.2008.18.1891

75. Phan C, Mindrum M, Silverman C, Paris K, Spanos W. Matchedcontrol retrospective study of the acute and late complications in patients with collagen vascular diseases treated with radiation therapy. Cancer J. 2003;9(6):461-466. doi:10.1097/00130404200311000-00005

76. Chen AM, Obedian E, Haffty BG. Breast-conserving therapy in the setting of collagen vascular disease. Cancer J. 2001;7(6):480-491.

77. Holmes D, Colfry A, Czerniecki B, et al. Performance and practice guideline for the use of neoadjuvant systemic therapy in the management of breast cancer. Ann Surg Oncol. 2015;22(10):3184-3190. doi:10.1245/s10434-015-4753-3

78. Barker AD, Sigman CC, Kelloff GJ, Hylton NM, Berry DA, Esserman LJ. I-SPY 2: an adaptive breast cancer trial design in the setting of neoadjuvant chemotherapy. Clin Pharmacol Ther. 2009;86 (1):97-100. doi:10.1038/clpt.2009.68

\section{Publish your work in this journal}

Breast Cancer - Targets and Therapy is an international, peer-reviewed open access journal focusing on breast cancer research, identification of therapeutic targets and the optimal use of preventative and integrated treatment interventions to achieve improved outcomes, enhanced survival and quality of life for the cancer patient.
The manuscript management system is completely online and includes a very quick and fair peer-review system, which is all easy to use. Visit http://www.dovepress.com/testimonials.php to read real quotes from published authors. 